\title{
A TAXA DE CÂMBIO REAL DE EQUILÍbRIO NO BRASIL*
}

\author{
Pablo Camacho Badani ${ }^{\S}$ \\ Álvaro Barrantes Hidalgo ${ }^{\alpha}$
}

\section{RESUMO}

O objetivo deste estudo é estimar a Taxa de Câmbio Real de Equilíbrio (TCRE) no Brasil e compará-la com a Taxa de Câmbio Real (TCR) observada para o período recente. A fim de estimar a TCRE, adotouse a metodologia desenvolvida inicialmente por Sebastian Edwards. Os dados são mensais e abrangem o período entre julho de 1994 (momento da implementação do Plano Real) até dezembro de 2002. Os resultados mostram que no momento da implementação do Plano Real a Taxa de Câmbio Real encontravase valorizada. De 1995 a 1998, os resultados mostram que a TCR estava próxima do seu nível de equilíbrio. A partir de 1999 até o final do período de análise, os resultados apontam uma desvalorização do câmbio real.

Palavras-chave: taxa de câmbio real de equilíbrio, desalinhamento da taxa de câmbio, Plano Real.

\section{ABSTRACT}

The object of this study is to estimate the Equilibrium Real Exchange Rate (ERER) for the Brazilian economy and compare it with the actual Real Exchange Rate (RER). To estimate the ERER, this study adopts the methodology developed initially by Sebastian Edwards. The estimation is based on monthly data, starting on the date of introduction of the Real Plan (July of 1994) to December of 2002. The results show that on the occasion of the introduction of the Real Plan the RER was over-valuated. From 1995 to 1998 the results show that the real exchange rate was near to its equilibrium level. From 1998 the results indicate a Real Exchange Rate that is under-valued.

Key words: equilibrium real exchange rate, exchange rate misalignment, Real Plan.

JEL classification: F31.

\footnotetext{
Os autores agradecem os comentários e sugestões valiosas recebidas dos pareceristas anônimos desta revista, isentando-os de quaisquer erros e omissões remanescentes.

$\S \quad$ Mestre em Economia pela Universidade Federal de Pernambuco - PIMES e Coordenador de Projetos no Instituto de Pesquisa Cultural para a Educação Popular (INDICEP) - Cochabamba, Bolívia.

a Doutor em Economia pela USP - SP, Professor do Departamento de Economia da UFPE e Pesquisador do CNPq. 


\section{INTRODUÇÃO}

É importante, para as autoridades econômicas e pesquisadores, conhecer a Taxa de Câmbio Real de Equilíbrio (TCRE), posto que o desalinhamento da Taxa de Câmbio Real (TCR) pode gerar problemas macroeconômicos que podem provocar efeitos negativos em variáveis tais como a inflação, o nível de emprego, a absorção, a alocação de recursos na economia etc. Portanto, o estudo da TCR é indispensável para um melhor entendimento e desenho das políticas econômicas pertinentes.

No Brasil, diversos trabalhos já foram realizados com vistas a conhecer melhor a TCRE. Salazar e Carvalho (1989) utilizaram a metodologia de Roe e Grenne (1986) para estimar a taxa de câmbio de equilíbrio para o período de 1960 a 1983. Tal metodologia define o valor da taxa de câmbio, para um país pequeno, como sendo aquela que mantém o equilíbrio na conta corrente sem a intervenção do governo. Trata-se de um modelo de determinação da taxa de câmbio de equilíbrio parcial e estático, em que também se supõe a ausência de movimentos de capitais. Os resultados mostraram que houve valorização do câmbio no período considerado. Gonzaga e Terra (1997) estudaram o efeito da volatilidade da taxa de câmbio real sobre o comércio usando um modelo de equilíbrio geral. Os autores constataram que a volatilidade da inflação do período 1979-1995 poderia ser considerada como uma das principais fontes de volatilidade da taxa de câmbio real no Brasil. Bonomo e Terra (1999) apresentam uma análise histórica da política cambial do Brasil, identificando os regimes cambiais de 1964 a 1997. No balanço histórico acerca da política cambial no Brasil, os autores identificaram ciclos eleitorais e encontraram indícios de a probabilidade de valorização da taxa de câmbio ser maior em meses que antecedem às eleições, enquanto que a probabilidade de uma taxa de câmbio desvalorizada é maior em meses posteriores às eleições. Melo (1998) apresenta um modelo de taxa de câmbio de equilíbrio denominada "taxa virtual", utilizada na análise de viabilidade de projetos. Segundo o autor, a taxa de câmbio virtual é capaz de refletir distorções resultantes da aplicação de políticas subótimas de dispêndio agregado, em vez de se ater apenas às distorções causadas pelo protecionismo comercial. As estimações do autor mostram que a taxa de câmbio parece ter estado continuamente valorizada no período de 1975-1995. Holanda (2002) estima a taxa de câmbio real de equilíbrio brasileira para o período 1975-1998. O autor utiliza três modelos alternativos. O primeiro baseado na Paridade do Poder de Compra, o segundo, no conceito de câmbio fundamental e um terceiro modelo denominado câmbio estrutural. Segundo o autor, os três modelos apontam a década de 1980 como de freqüente subvalorização cambial. Os inícios das décadas de 1980 e 1990 parecem ser períodos de equilíbrio. Após o Plano Real há um período de forte sobrevalorização, que vai sendo gradualmente eliminada a partir do segundo trimestre de 1995. Por outro lado, a crise financeira de 1997 levou a uma sobrevalorização do câmbio real da ordem de 15\% em 1998. Merlin e Portugal (2002) estimaram a taxa de câmbio real de equilíbrio no Brasil no período 1984-1999. Os autores estimam coeficientes de longo prazo de um modelo de cointegração. Os resultados indicam que os fundamentos da economia geraram uma tendência de redução do desalinhamento cambial pós-1994.

Apesar do debate sobre a TCRE, a evidência empírica parece mostrar uma valorização da taxa de câmbio no Brasil desde meados da década de 1980. O objetivo deste artigo é analisar os possíveis desalinhamentos da taxa de câmbio a partir da implementação do Plano Real. Para isso será estimada a TCRE para a economia brasileira, e esta será comparada com a TCR observada em cada período. O propósito do artigo é fornecer subsídios para a discussão sobre o desalinhamento cambial no Brasil no período pós-Plano Real. Para alcançar este objetivo realizaremos a estimação da TCRE utilizando a metodologia desenvolvida por Edwards (1988). Assim, após esta introdução, 
será apresentado o modelo utilizado na estimação empírica, seguido da apresentação dos resultados obtidos e, por último, as conclusões do trabalho.

\section{MODELO PARA A ESTIMAÇÃO EMPÍRICA DA TCRE}

A estimação da TCRE será realizada por meio do uso do modelo intertemporal proposto por Edwards (1988). Trata-se de um modelo do setor real de uma economia pequena, aberta e que leva em conta o governo. Além de incorporar diferentes períodos de tempo, o modelo leva em consideração os fundamentos microeconômicos. O modelo não contempla variáveis monetárias, portanto, a taxa de câmbio nominal é irrelevante e não interessa o sistema cambial vigente. No modelo, a taxa de câmbio é definida como sendo o preço relativo entre bens comercializáveis e não comercializáveis. O modelo que servirá de base para estimação da TCRE pode ser expresso, na forma reduzida, da seguinte forma:

$\log \operatorname{TCRE}_{t}=\beta_{0}+\beta_{1} \log (T T)_{t}+\beta_{2} \log (\operatorname{SPREAD})_{t}+\beta_{3} \log (\mathrm{CONCAP})_{t}$

$+\beta_{4} \log (G G N)_{t}+\beta_{5} \log (\text { INVPIB })_{t}+\beta_{6} \log (\text { PROGTEC })_{t}+u_{t}$

onde, como definido acima, TCRE é a Taxa de Câmbio Real de Equilíbrio, (TT) são os termos de troca; $(S P R E A D)$ é um índice de abertura do país e que pretende levar em conta os efeitos dos controles de câmbio e as restrições ao comércio internacional; (CONCAP) representa as restrições aos fluxos de capitais; $(G G N)$ representa o gasto do governo em bens não comercializáveis; (INVPIB) é o investimento como porcentagem do PIB, e representa a acumulação de capital; (PROGTEC) é uma medida que pretende considerar os efeitos do progresso tecnológico; por último u é a perturbação aleatória. Na equação (1) a TCRE é determinada pela tendência de uma série de variáveis reais denominadas "fundamentos". Estas variáveis são tanto de natureza externa quanto de natureza interna.

A estimação da equação (1) apresenta alguns problemas metodológicos, uma vez que não é possível estimá-la econometricamente, dado que a TCRE não é observada em cada período de tempo. No entanto, é possível observar a TCR em cada período e, com base nesta, pode-se estimar a TCRE.

A TCR observada responde tanto aos "fundamentos" como às variáveis monetárias, e pode encontrar-se ou não fora do seu nível de equilíbrio. Portanto, é necessário utilizar um modelo que incorpore estas variáveis e mostre o comportamento dinâmico da TCR para, desta forma, obter uma boa estimação da TCR observada. Esta variável pode apresentar desequilíbrios devido a mudanças nos fundamentos ou pelo efeito de políticas macroeconômicas. A dinâmica da TCR pode ser representada por meio de uma equação que explique tais movimentos:

$\left(\log T C R_{t}-\log T C R_{t-1}\right)=\theta\left(\log T C R E_{t}-\log T C R_{t-1}\right)-a\left(Z_{t}-Z^{*}\right)+b\left(\log E_{t}-\log E_{t-1}\right)$

As variáveis $T C R$ e $T C R E$ foram acima definidas, sendo a TCRE uma função dos fundamentos (ver equação (1)); $Z_{t}$ é um vetor que mede as políticas macroeconômicas (por exemplo, o crescimento da oferta de dinheiro); $Z^{*}{ }_{t}$ é o nível de políticas macroeconômicas consistentes com o equilíbrio; e $E$ é a taxa de câmbio nominal. Os parâmetros " $\theta$ ", " $a$ " e " $b$ " capturam os aspectos mais importantes da dinâmica do processo de ajuste.

O lado esquerdo da equação (2) representa as variações na TCR observada. Por sua vez, o lado direito mostra que estas mudanças respondem a três forças. Primeiro, existirá uma tendência 
natural de a TCR corrigir o desequilíbrio, dado que os preços dos bens não comercializáveis tenderão a se mover em direção ao equilíbrio. Isto é representado por meio da expressão $\theta\left(\log T C R E_{t}\right.$ $\left.\log T C R_{t-1}\right)$, onde $\theta$ é a velocidade do ajuste. O parâmetro $\theta$ é um número entre zero e a unidade, e quanto menor for o parâmetro $\theta$, mais lento será o ajuste; caso contrário, se o valor do $\theta$ é igual a 1 , isto significa que qualquer desvio produzido na $T C R$, este será corrigido no período seguinte. Em geral, se existir valorização, o processo será mais lento. O segundo elemento de (2) se refere às políticas macroeconômicas representadas pelo vetor $a\left(Z_{t}-Z^{*}{ }_{t}\right)$. Quanto mais longe estiver $Z_{t}$ de $Z^{*}{ }_{t}$, $\left(Z_{t}>Z^{*}\right)$, maior será a tendência da TCR se valorizar.

Por último, inclui-se o efeito da desvalorização da taxa de câmbio nominal, que está representado pela expressão $b\left(\log E_{t}-\log E_{t-1}\right)$. De acordo com esta expressão, as desvalorizações nominais terão um efeito positivo sobre a $T C R$, gerando um aumento nesta. Com essa informação é possível formular um modelo para estimá-lo econometricamente, substituindo, inicialmente, a equação (1) em (2), obtendo-se a seguinte expressão:

$\log T C R_{t}=\alpha_{0}+\alpha_{1} \log (T T)_{t}+\alpha_{2} \log (S P R E A D)_{t}+\alpha_{3} \log (C O N C A P)_{t}+\alpha_{4} \log (G G N)_{t}$

$+\alpha_{5} \log (I N V P I B)_{t}+\alpha_{6} \log (\text { PROGTEC })_{t}+(1-\theta) \log T C R_{t-1}-a\left(Z_{t}-Z^{*}\right)+b(D E V A L)_{t}+U_{t}$

onde $\alpha_{i}=\beta i \theta$ (para todo $i$ ), DEVAL representa as desvalorizações nominais e $U$ é o termo de perturbação aleatório. A equação (3) descreve o comportamento da TCR observada, como uma combinação do efeito das variáveis fundamentais e as políticas macroeconômicas.

\section{ESTIMAÇÃO DA TAXA DE CÂMBIO REAL DE EQUILÍBRIO}

Com base nos parâmetros obtidos da estimação econométrica da equação (3) pode-se gerar coeficientes para se chegar às séries estimadas da TCRE. Mencionou-se anteriormente que a TCRE é uma tendência de longo prazo, e que está determinada estritamente por seus "fundamentos". Portanto, é necessário considerar uma situação de equilíbrio em que o setor monetário esteja equilibrado. Isto significa impor as restrições (na equação 3) de que " $a$ ” e " $b$ ” são iguais a zero, ou seja, as políticas macroeconômicas e as desvalorizações nominais não possuem nenhum efeito sobre a $T C R E$, e no momento da estimação assume-se que estas estão nos seus valores de tendência, ou seja, $Z_{t}=Z^{*}{ }_{t}$ e $D E V A L=0$.

Uma vez imposta esta restrição, calculam-se os coeficientes de longo prazo $\left(\beta_{i}\right)$ da TCRE. Para isso, dividem-se os $\alpha_{i}$ entre $\theta$, onde os $\alpha_{i}$ são os parâmetros para os "fundamentos" obtidos na equação (3) e $\theta$ é a velocidade de ajuste endógena, obtida do coeficiente da TCR defasada um período, na mesma equação. Desta forma, obtêm-se os coeficientes $\beta_{i}$ da equação da TCRE necessários para gerar séries estimadas da TCRE.

No momento de se estimar as séries da TCRE surge o problema de saber quais valores dos "fundamentos" devem ser utilizados para efetuar o cálculo. A melhor alternativa é elaborar uma "média móvel" destes valores. Esta técnica tem a propriedade de "suavizar" as mudanças abruptas que possam se apresentar nas variáveis. Desta maneira, as mudanças que se apresentarem na $T C R E$ serão um reflexo de mudanças estruturais reais e não de desequilíbrios transitórios. 


\section{COMPARAÇÃo ENTRE A TAXA DE CÂMBIO REAL OBSERVADA E A DE EQUILÍBRIO}

A finalidade da geração de séries estimadas tanto da TCR observada quanto da TCRE é comparar ambas as séries. Desta forma será possível observar o comportamento de ambas e estabelecer os possíveis desalinhamentos. Em outras palavras, poder-se-á estabelecer se a TCR no Brasil, a partir da implementação do Plano Real (Julho de 1994), encontra-se ou não fora do seu nível de equilíbrio. Simultaneamente, será possível analisar as causas destes desalinhamentos e o efeito das políticas macroeconômicas no comportamento da TCR.

A fim de estabelecer os desvios ao comparar as séries estimadas de ambas as TCRs é necessário "ancorá-las”, ou seja, deve-se assumir que em algum momento no tempo a TCR efetiva encontrava-se no seu nível de equilíbrio. Somente assim será possível identificar a presença de desalinhamentos, e poder-se-á quantificar a magnitude das desvalorizações ou supervalorizações da TCR. Para o caso do Brasil, assume-se como ponto de "ancoragem" setembro de 1994. Esta decisão baseia-se no resultado do estudo de Holanda (2002), que encontrou que no momento da introdução do Plano Real a taxa de câmbio real estava numa situação próxima ao equilíbrio.

\section{DEFINIÇÃo DE VARIÁVEIS DE APROXIMAÇÃo PARA O CASO DO BRASIL}

a) Taxa de Câmbio Real (TCR). Utilizar-se-á como variável representativa do preço dos bens comercializáveis o índice de preços ao consumidor nos EUA (dado que esses preços evoluem de acordo com a inflação internacional), e como proxy do preço dos bens não comercializáveis o índice de preços ao consumidor nacional (dado que este contém, em sua maioria, preços de bens não comercializáveis, tais como moradia, serviços de transporte, serviços diversos etc.). Assim, temos que:

$$
T C R=\frac{E \cdot(I P C E U)}{I P C B R A}
$$

$\mathrm{Na}$ expressão (4) foi utilizado o índice de preços ao consumidor dos EUA, tendo em vista a importância desse país na economia mundial e no comércio exterior brasileiro. Uma alternativa mais abrangente consiste em utilizar um índice de preços ponderado dos IPCs dos principais sócios comerciais do Brasil. A taxa de câmbio real calculada utilizando os IPC de vários países é chamada de taxa de câmbio efetiva.

b) Termos de Troca (TT). O índice desta variável será calculado dividindo-se o índice de preços de exportação pelo índice de preços de importação.

c) Controles de Câmbio (SPREAD). No presente estudo, define-se como proxy desta variável o "spread" ou a diferença entre as cotações da taxa de câmbio oficial e a taxa de câmbio no mercado paralelo. "Esta variável captura num sentido amplo a severidade dos controles de câmbio e as restrições ao comércio internacional." (Edwards, 1988). ${ }^{1}$

d) Controles dos Fluxos de Capital (CONCAP). A quantificação do fluxo líquido de capital será realizada da seguinte maneira: CONCAP $=$ Entrada de capitais estrangeiros - Saída de capitais estrangeiros.

A entrada de capital estrangeiro é composta por investimento direto e de carteira, desembolsos da dívida externa e transferências unilaterais, enquanto que a saída compreende os serviços da dí-

1 A mesma variável de aproximação foi utilizada em Edwards (1988) e Holanda (2002, p. 694). 
vida externa. Para evitar problemas de endogeneidade desta variável no modelo proposto, esta será defasada um período. Assim, o fluxo de capital já terá ocorrido no momento de determinar a TCR e esta será afetada pela entrada de capitais e não o inverso.

e) Gasto do Governo em Bens Não-Comercializáveis (GGN). Para esta variável não existem dados que mostrem o gasto do governo desagregado entre bens comercializáveis e bens não comercializáveis. Portanto, utilizaremos como variável de aproximação os gastos correntes do governo. A escolha deste gasto baseia-se na suposição de que a maior parte destas despesas é destinada principalmente à aquisição de bens não comercializáveis. No caso do Brasil, isto parece razoável, dado que a maior parte destes gastos se destinam a salários, saúde, educação, moradia, infra-estrutura, que podem ser considerados como gastos em bens não comercializáveis.

f) Investimento como Porcentagem do PIB (INVPIB). Para construir uma série mensal desta variável depara-se com o problema de somente existirem dados trimestrais dessa relação. Para superá-lo assumir-se-á que esta relação se manteve constante no trimestre. Desta forma, é possível construir uma série mensal usando os dados trimestrais como variável de controle.

g) Progresso Tecnológico (PROGTEC). Para esta variável, que reflete os ganhos de produtividade, será utilizado como aproximação o valor defasado das importações de maquinarias e equipamentos. ${ }^{2}$ Admite-se aqui que a importação desses bens contribuem para a melhoria da produtividade.

h) Políticas Macroeconômicas. Para a análise do caso do Brasil serão tomadas como indicadores da política econômica duas variáveis. A primeira será a taxa de crescimento da oferta monetária (CREM1), medida por meio do crescimento de M1. Isto, para analisar o possível efeito da política monetária no comportamento da TCR. A segunda variável representará a influência da política fiscal, e consistirá na razão entre o déficit fiscal e a emissão monetária (DEFEM), sendo possível, dessa maneira, estabelecer se a gestão da política fiscal tem contribuído ou não para gerar pressões inflacionárias e, portanto, se esta tem afetado a TCR.

i) Desvalorizações Nominais (DEVAL). Para esta variável utilizar-se-á a desvalorização da taxa de câmbio nominal oficial, isto tendo em vista que as transações de comércio exterior são realizadas com base na taxa de câmbio nominal.

Os dados necessários para construir as séries mensais das variáveis de aproximação para o Brasil foram obtidos do Banco Central do Brasil (BACEN) e do Instituto de Pesquisa Econômica Aplicada (IPEA), por meio de suas respectivas páginas na internet: www.bacen.gov.br e www.ipea.gov.br. As páginas foram acessadas de dezembro de 2002 a março de 2003.

\section{SINAIS ESPERADOS DOS COEFICIENTES}

Os sinais esperados dos coeficientes, de acordo com a teoria, serão descritos em seguida.

Uma queda dos termos de troca gera um efeito renda negativo, ou seja, reduz a renda real. Esta diminuição na renda real ocasionará uma menor demanda por bens não comercializáveis, e para restabelecer o equilíbrio seu preço deve diminuir, ocasionando, deste modo, um aumento da TCR. Contudo, deve-se considerar que esta queda nos termos de troca também pode gerar um efeito substituição no consumo de bens e originar um incremento na demanda de bens não comercializáveis, ocasionando um aumento no seu preço e, portanto, valorizando a TCR. Por esta razão, não se pode conhecer, a priori, a reação da TCR diante de uma deterioração nos termos de troca.

2 A mesma variável de aproximação foi utilizada por Holanda (2002, p. 693). 
No caso de controles dos fluxos de capital, um relaxamento dos mesmos se traduzirá num incremento na entrada de capital e de crédito externo. Conseqüentemente, será elevado o nível de gasto corrente que incrementará, em parte, a demanda por bens não comercializáveis. Como resultado, e para manter o equilíbrio, haverá um aumento no preço dos bens não comercializáveis, provocando, deste modo, uma valorização da TCRE. Por outro lado, caso seja permitido que a taxa de câmbio nominal varie, a maior entrada de capitais (ou seja, maior oferta de dólares) diminuirá este valor, acelerando a valorização da TCR. Os efeitos das taxas de juros mundiais, e das transferências e ajuda internacional, são similares ao caso anterior.

O nível e a composição do gasto público é outra variável que afeta a TCR. A forma como esta será afetada dependerá de como o gasto entre bens comercializáveis e não comercializáveis será distribuído. Se a totalidade ou a maior parte do gasto for destinada à compra de bens não comercializáveis, a demanda por estes bens será incrementada, fazendo subir seu preço, causando, assim, uma valorização da TCR. O contrário ocorrerá se o gasto se concentrar na compra de bens comercializáveis.

O efeito do progresso tecnológico sobre a TCR tem sido amplamente discutido na literatura internacional. Balassa (1964) e Samuelson (1964) identificaram um padrão de comportamento da TCR baseado no aumento diferenciado da produtividade. As evidências internacionais parecem mostrar que as diferenças de produtividade são maiores nos bens comercializáveis do que nos bens não comercializáveis. Países ricos com altas taxas de crescimento de produtividade no setor de bens comercializáveis tendem a ter preços mais elevados dos bens não comercializáveis e níveis gerais de preços também mais elevados. Esses países apresentam apreciação real de suas moedas. ${ }^{3}$ Por outro lado, caso o aumento da produtividade seja o mesmo nos dois setores, comercializáveis e não comercializáveis, e supondo que o aumento da oferta predomine sobre o aumento da demanda, então espera-se efeito positivo sobre a TCR, ou seja, uma desvalorização. Na economia brasileira, o processo de abertura comercial, a privatização, a desregulamentação da atividade econômica e os programas de qualidade, implementados na década de 1990, levaram a um expressivo crescimento da produtividade na indústria. ${ }^{4}$ Assim, a manutenção, por parte do governo, de uma taxa de câmbio que sobrevalorizava o real ante o dólar, após o Plano Real, parecia ser suportável por parte dos agentes num contexto de elevado crescimento de produtividade.

O nível de investimento interno também afeta a TCR, dado que influi na acumulação de capital. Quanto maior for a porcentagem de investimento em relação ao PIB, maior será a oferta tanto de bens comercializáveis quanto de não comercializáveis. Sendo assim, o preço dos comercializáveis permanece constante porque este é fixado internacionalmente, mas o preço dos não comercializáveis deve diminuir, ocasionando um aumento no nível da TCR e, desta forma, uma desvalorização.

3 Bhagwati (1984) e Kravis e Lipsey (1983) desenvolveram uma análise alternativa para explicar os níveis maiores de preços dos países ricos, baseado nas diferenças nas dotações de capital e trabalho. Como os países ricos têm relações capital-trabalho mais elevadas, eles terão salários e preços dos bens não comercializáveis também mais elevados. A diferença internacional no preço relativo dos bens não comercializáveis sugere níveis gerais de preços, medidos em uma única moeda, mais elevados nos países ricos do que nos países pobres e, portanto, uma apreciação real de suas moedas.

4 As evidências mostram que a produtividade total dos fatores, ajustada para as economias de escala e as margens de mark-up, passou de uma média de $0,5 \%$ ao ano antes da reforma, para 3,3\% ao ano após a introdução da reforma comercial. Ver Hidalgo (2002). 


\section{ESTIMAÇÃO DA TAXA DE CÂMBIO REAL NO BRASIL}

Dado que as variáveis do modelo não são da mesma ordem, não é possível utilizar a metodologia de co-integração. Por esse motivo, adotar-se-á a metodologia de Mínimos Quadrados Ordinários (MQO). A equação a ser estimada é a seguinte:

$$
\begin{aligned}
& \text { LTCR }_{t}=\alpha_{0}+\alpha_{1}(T T)_{t}-\alpha_{2}(\text { SPREAD })_{t}-\alpha_{3}(\text { CONCAP })_{t}-\alpha_{4}(G G N)_{t}+\alpha_{5}(\text { INVPIB })_{t}+ \\
& \alpha_{6}(\text { PROGTEC })_{t}+(1-\theta) \text { LTCR }_{t-1}-\mathrm{a}_{1}(C R E M 1)-\mathrm{a}_{2}(\text { DEFEM })+\mathrm{b}(\text { DEVAL })_{t}+u_{t}
\end{aligned}
$$

A equação (5) representa os movimentos de curto prazo da TCR. O coeficiente $(1-\theta)$, da variável $L T C R_{t-1}$, será utilizado para estimar os coeficientes $\beta_{i}$ necessários para construir a série estimada da Taxa de Câmbio Real de Equilíbrio $\left(\beta_{i}=\frac{\alpha_{i}}{\theta}\right)$. Note-se que a única diferença da equação (5) para a equação (3), da seção anterior, é que o termo $a\left(Z_{t}-Z^{*}{ }_{t}\right)$, em (3), foi dividido nas duas variáveis de aproximação que representam a política econômica do governo: DEFEM e CREM1.

Tabela 1 - Resultados econométricos - coeficientes de curto prazo - variável dependente: LTCR

\begin{tabular}{lcccc}
\hline Variável Proxy & Coeficiente Estimado & Erro Padrão & T-Ratio & P-Value \\
\hline TT & $-2,51 \mathrm{E}-03$ & $1,31 \mathrm{E}-03$ & $-1,91$ & 0,058 \\
INVPIB & $8,94 \mathrm{E}-03$ & $4,06 \mathrm{E}-03$ & 2,2 & 0,03 \\
PROGTEC & $4,69 \mathrm{E}-05$ & $2,35 \mathrm{E}-05$ & 1,99 & 0,049 \\
DEFEM & $-1,6$ & $5,75 \mathrm{E}-07$ & $-2,55$ & 0,012 \\
CREM1 & $-1,47 \mathrm{E}-06$ & $4,11 \mathrm{E}-02$ & $-2,52$ & 0,013 \\
DEVAL & $-0,10359$ & $3,05 \mathrm{E}-02$ & 0 \\
LTCR $_{\mathrm{t}-1}$ & 0,41823 & $3,62 \mathrm{E}-02$ & 13,74 & 0 \\
CONSTANT & 0,90917 & 0,153 & 25,08 & 0,697 \\
\hline $\mathrm{F}=643,012$ & $5,97 \mathrm{E}-02$ & & 0,39 & \\
\hline
\end{tabular}

$(L M)=0,339$

Fonte: Cálculos dos autores.

Note-se também em (5) que para a estimação empírica do modelo adotou-se um modelo semi-log. A partir daí foi necessário identificar o melhor ajuste. O diagnóstico dos modelos estimados foi realizado por meio das estatísticas usuais de exame de ajuste $\left(R^{2}\right)$ e significância dos parâmetros (estatísticas $t$ e $F$ ). Para a escolha entre modelos alternativos foram utilizados os critérios mais comuns: Akaike Information Criterion (AIC) e Schwartz Bayesian Criterion (SBC). O princípio da parcimônia também foi levado em conta no momento de identificar o melhor ajuste, isto é, eliminando variáveis que se mostraram não significativas. Além disto, não há risco de se incorrer no erro de especificação no modelo, dado que os fundamentos da taxa de câmbio descritos na teoria podem resultar significativos para um país e não para outros. Os coeficientes estimados das variáveis SPREAD, GGN e CONCAP não resultaram estatisticamente significativos, portanto, estas fo- 
ram eliminadas. Assim, o melhor ajuste foi obtido ao eliminar estas três variáveis e defasando 6 períodos (ou seja, dois trimestres) a variável DEFEM. Nas estimações foram utilizados dados mensais a fim de se levar em conta possíveis flutuações da TCR ao longo do ano. O período de análise vai de janeiro de 1994 até dezembro de 2002. A Tabela 1 apresenta os resultados obtidos na estimação econométrica do modelo.

A estatística F utilizada na análise da variância indica que a regressão é estatisticamente significativa com um nível de significância de 95\%. A estatística do Multiplicador de Lagrange (LM) mostrou que não existe autocorrelação. Foi necessário usar essa estatística porque o modelo inclui um termo auto-regressivo. A significância estatística individual dos regressores foi avaliada mediante o teste " $t$ ". Os resultados são em geral satisfatórios. Observa-se que todos os coeficientes apresentam os sinais conforme o esperado e são significativos a $95 \%$ de confiança. O coeficiente da variável TT resultou significativo a 94,2\% de significância.

Observa-se na Tabela 1 que o coeficiente $\mathrm{R}^{2}$ e $\mathrm{R}^{2}$ ajustado são bastante próximos. Este resultado mostra que as variáveis explicativas incluídas no modelo são suficientes, ou seja, não é necessário incluir (ou excluir) qualquer variável explicativa ao modelo. Como indicado acima, não é possível determinar a priori o sinal da variável "Termos de Troca". Estudos anteriores sugerem que o sinal desta variável é negativo. ${ }^{5} \mathrm{Na}$ estimação realizada neste trabalho o coeficiente resultou negativo e estatisticamente significativo, indicando que a deterioração dos termos de troca no Brasil se traduz em uma desvalorização da TCR. Portanto, pode-se concluir que, no Brasil, o efeito renda é o predominante. Este resultado implica que uma deterioração nos termos de troca gera um efeito renda negativo, reduzindo a renda real. Esta redução da renda real se traduz em uma menor demanda de bens não comercializáveis e, portanto, para restabelecer o equilíbrio o preço destes bens diminui, gerando (por meio da relação $T C R=\frac{P_{T}}{P_{N}}=\frac{E \cdot P_{T}^{*}}{P_{N}}$ ) uma desvalorização da TCR.

A variável utilizada para aproximar o progresso tecnológico merece comentários. O incremento na produtividade, resultante do progresso tecnológico, provoca um aumento na oferta, e os preços dos bens não comercializáveis tendem a diminuir. Simultaneamente, o efeito renda, como conseqüência da maior produtividade, ocasiona um incremento na demanda e, portanto, no preço. Também, como indicado acima, o aumento da produtividade pode não ser o mesmo nos dois setores (comercializáveis e não comercializáveis). Os resultados empíricos encontrados parecem indicar que o aumento da oferta é o predominante. Portanto, supondo que a produtividade aumenta de igual forma em ambos os setores, um incremento na produtividade parece ter um efeito positivo sobre a TCR, ou seja, causa uma desvalorização. No que se refere à variável de aproximação que mensura o nível de investimento, esta resultou ser estatisticamente significativa e com sinal positivo, o que indica que um maior nível de investimento ocasionará uma desvalorização da TCR e vice-versa.

O efeito esperado das políticas macroeconômicas, representadas pelas variáveis CREM1 e DEFEM, resultaram negativos conforme a teoria e foram estatisticamente significativos. As variáveis relacionadas com as políticas do governo (CREM1 e DEFEM) parecem afetar negativamente a TCR. Contudo, os coeficientes relativamente pequenos $(-0,10359$ e $-0,14668 \mathrm{E}-05$, respectivamente) mostram que se políticas domésticas inconsistentes com o equilíbrio forem mantidas por períodos muito longos estas podem gerar desequilíbrios substanciais.

5 No estudo de Holanda (2002) o sinal resultante da variável Termos de Troca foi negativo e estatisticamente significativo. O mesmo resultado foi obtido por Edwards (1988) em um estudo do comportamento da TCR em 12 países em desenvolvimento. 
O coeficiente da variável que representa as desvalorizações nominais resultou positivo e estatisticamente significativo. A teoria indica, por meio da relação $T C R=\frac{P_{T}}{P_{N}}=\frac{E \cdot P_{T}^{*}}{P_{N}}$, que uma desvalorização, ou seja, um aumento na taxa de câmbio nominal $(E)$, leva a um aumento ou desvalorização da TCR.

A velocidade de ajuste endógena do sistema (o valor desse parâmetro é obtido do coeficiente da TCR defasado um período), conforme a teoria, é um número positivo entre zero e um. Observa-se, na Tabela 1, que o coeficiente estimado é 0,90917 , portanto, a velocidade de ajuste do sistema é 0.09. Isto indica que, na ausência de intervenção, o ajuste automático é bastante lento, dado que, em média, $9 \%$ de um desvio dado da TCR será corrigido em um período. Este resultado não é surpreendente, visto que em Holanda (2002) a velocidade de ajuste para o Brasil também mostrou a mesma lentidão, corrigindo-se aproximadamente $14 \%$ do desalinhamento em cada trimestre. (Holanda 2002, p. 696).

\section{ESTIMAÇÃO DA TAXA DE CÂMBIO REAL DE EQUILÍBRIO NO BRASIL}

Com base nos coeficientes das regressões é possível obter os parâmetros necessários para construir a série da TCRE. O primeiro passo consiste em eliminar todas as variáveis monetárias do modelo (CREM1, DEFEM e DEVAL), dado que a TCRE somente responde a variáveis reais. Posteriormente, dividem-se os parâmetros obtidos na regressão da equação (5) pela estimativa da velocidade de ajuste automático do sistema $(\theta=0,09)$. Na Tabela 2 apresentam-se os parâmetros necessários para construir a série estimada da TCRE.

Tabela 2 - Resultados econométricos - coeficientes de longo prazo

\begin{tabular}{lc}
\hline Variável & Coeficiente \\
\hline CONSTANTE & 0,6577893 \\
TT & $-0,0276362$ \\
INVPIB & 0,0984565 \\
PROGTEC & 0,0005163 \\
\hline
\end{tabular}

Fonte: cálculos dos autores.

O passo seguinte foi construir a série da TCRE utilizando uma média móvel (de seis períodos) das variáveis explicativas. Este procedimento parece ser o mais adequado para refletir as tendências de longo prazo consistentes com o equilíbrio. ${ }^{6}$ Por último, foi construído o índice com base em setembro de 1994, assumindo que ambas as TCRs tinham o mesmo valor naquele período. Já foi apontado que a decisão de ancorar as séries nesta data baseia-se no estudo de Holanda (2002, p. 691), no qual se observa que no terceiro trimestre de 1994 a TCR encontrava-se próxima do equilíbrio. O Gráfico 1 apresenta os resultados obtidos dessas estimações para o caso do Brasil.

6 Nos estudos de Holanda (2002) e Edwards (1988), foram utilizadas médias móveis de 2 trimestres, ou seja, 6 meses. 


\section{Gráfico 1 - Análise dos desalinhamentos da taxa de câmbio no Brasil}

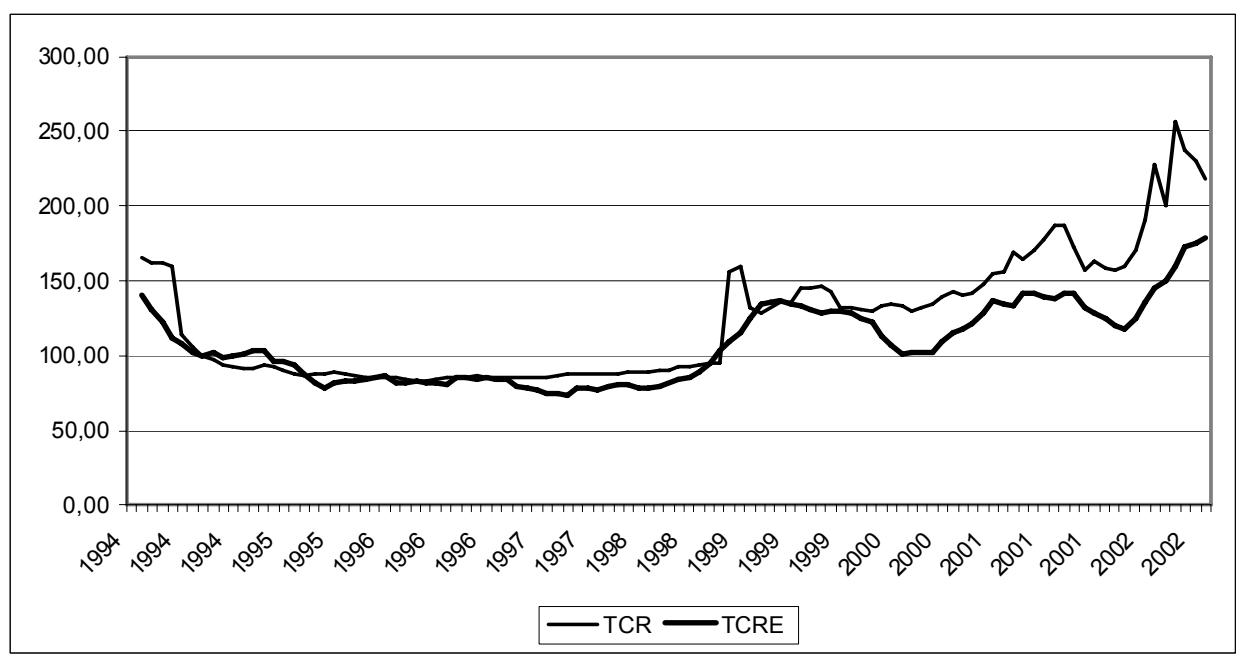

Fonte: Cálculos dos autores.

No Gráfico 1 podem ser observados 4 períodos com características similares. Inicialmente, no período anterior ao Plano Real, o câmbio real encontrava-se desvalorizado. Situação similar à encontrada no estudo de Merlin e Portugal (2002) e no estudo de Holanda (2002). De setembro de 1994 até abril de 1995, logo após à implementação do Plano Real, a TCR mostra sinais de valorização. Este resultado é semelhante ao obtido no estudo de Holanda (2002), com a diferença de que, neste último, a valorização da TCR perdura até o primeiro semestre de 1996. O autor observa uma valorização do câmbio real a partir do primeiro trimestre de 1995 até o terceiro trimestre de 1996. O resultado obtido por Merlin e Portugal, neste subperíodo, aponta também para uma valorização no câmbio real. A partir de maio de 1995 até dezembro de 1998, a TCR mostra pequenos desalinhamentos de curto prazo, podendo-se concluir que neste subperíodo a TCR estava próxima do equilíbrio. O estudo de Holanda (2002) também encontra uma situação próxima a esta no final de 1996 até o primeiro trimestre de 1997. Neste subperíodo, o estudo de Merlin e Portugal continua mostrando valorização no câmbio real.

O período janeiro de 1999 até o final do período em análise constitui-se um período de crescente desvalorização da TCR, situação que evolui de persistentes desvalorizações de curto prazo a uma desvalorização crônica, que começa em março de 2000 e perdura até o final do período em análise. O período da amostra estudada por Holanda (2002) vai somente até o terceiro trimestre de 1998, não obstante isto, os resultados apontam para uma crescente desvalorização a partir do segundo trimestre de 1997. Os resultados do estudo de Merlin e Portugal (2002), por sua vez, mostram que a taxa de câmbio real no Brasil permaneceu valorizada até dezembro de 1998. A partir do primeiro trimestre de 1999 até dezembro de 2000, os resultados apontam para uma desvalorização cambial.

A desvalorização no final do período em análise reflete principalmente a crise cambial ocorrida antes do primeiro turno das eleições presidenciais em outubro de 2002 e antes do segundo turno em novembro do mesmo ano. Observa-se que durante o período de vigência do sistema de bandas cambiais os desalinhamentos da TCR não foram tão severos nem tão persistentes como os resultantes no período de vigência da taxa flutuante. No começo do Plano Real a flutuação livre do câmbio real causou um forte e persistente desalinhamento negativo (valorização), e a partir da implantação 
do sistema cambial flutuante, em janeiro de 1999, a flutuação da taxa de câmbio provocou um desalinhamento positivo que perdurou até o final do período em análise.

Nas circunstâncias descritas, parece justificável a intervenção do governo para orientar e ajudar o mercado a encontrar um preço compatível com o equilíbrio de longo prazo.

\section{CONCLUSÕES}

Os resultados empíricos obtidos apontam a época anterior ao Plano Real como um período de desvalorização do câmbio real. Já o início do Plano Real - fase de vigência do regime cambial flutuante - caracterizou-se como um período de valorização cambial. Entre agosto de 1995 a dezembro de 1998 - período de vigência do sistema de bandas cambiais - pode ser considerado como um período onde a Taxa de Câmbio Real encontrava-se próxima do equilíbrio.

A comparação entre a TCRE e a TCR observada parece mostrar que a partir de 1999 inicia-se um período de crescente desvalorização da moeda nacional, situação que se aprofunda a partir de janeiro de 2000 até o final do período em análise. Encontra-se, portanto, um comportamento contrário ao previsto no estudo de Bonomo e Terra (1999), no qual os períodos pré-eleitorais seriam caracterizados por valorização cambial. Por outro lado, antes das eleições de 2002 a moeda brasileira apresenta uma forte desvalorização.

Os resultados obtidos neste trabalho são similares aos do estudo de Holanda (2002), ou seja, eles mostram que nos períodos de vigência do regime cambial flutuante a TCR apresenta fortes desalinhamentos em relação ao nível de equilíbrio. Por sua vez, no período de vigência do sistema de bandas cambiais a TCR encontrava-se próxima do equilíbrio.

A partir de janeiro de 1999 observa-se uma forte desvalorização cambial. Isto pode ser explicado principalmente pelo fato de o mercado de câmbio brasileiro responder fortemente a qualquer mudança no risco percebido pelos agentes. No entanto, se a atenção se concentrar nos períodos de vigência do sistema cambial flutuante, e simultaneamente considerar o início do Plano Real como um período de otimismo nas expectativas dos agentes e o final do período de análise como aquele de pessimismo nas expectativas dos agentes econômicos, os dados parecem mostrar que em períodos de otimismo na economia brasileira, o câmbio real tende a se valorizar, enquanto que em períodos de pessimismo, o câmbio real tende a se desvalorizar. Esta hipótese pode ser testada empiricamente em estudos posteriores que incluam na análise o período posterior às eleições no final de 2002.

\section{BIBLIOGRAFIA}

Balassa, Bela. The purchasing power parity doctrine: a reappraisal. Journal of Political Economy, 72, p. 584-596, 1964.

Bhagwati, Jagdish. Why are services cheaper in the poor countries? Economic Journal, 94, p. 279-286, 1984.

Bonomo, Marco; Terra, Cristina. The political economy of exchange rate policy in Brazil: 1964-1997. Revista Brasileira de Economia, n. 53, 1999.

Edwards, Sebastian. Real exchange rates, devaluation and adjustment: exchange rate policy in developing countries. Cambridge: MIT Press, 1988. 
Gonzaga, Gustavo; Terra, Cristina. Equilibrium real exchange rate, volatility, and stabilization. Journal of Development Economics, v. 54, p. 77-100, 1997.

Hidalgo, Álvaro B. O processo de abertura comercial brasileira e o crescimento da produtividade. Economia Aplicada, v. 6, n. 1, p. 79-95, jan-mar 2002.

Holanda, Marcos. O câmbio de equilíbrio no Brasil. Economia Aplicada, v. 6, n. 4, p. 681-701, out-dez 2002.

Kravis, Irving B.; Lipsey, Robert E. Toward an explanation of national price levels. Princeton Studies in International Finance 52, Department of Economics, Princeton University, 1983.

Melo, Fabiana. A taxa virtual: uma alternativa para a taxa de câmbio de equilíbrio. $22^{\circ}$ Prêmio BNDES de Economia, 1998.

Merlin, Carlos; Portugal, Marcelo. Estimating the equilibrium real exchange rate for Brazil: 1984-2000. Anais do XXX Encontro Nacional de Economia ANPEC. Nova Friburgo RJ, dezembro de 2002.

Roe, T.; Grenn, D. The estimation of a shadow equilibrium exchange rate: a direct method. In: Salazar, Antonio; Carvalho, José Luis, Uma estimativa da taxa de câmbio de equilíbrio de livre comércio para o Brasil: 1960-1983. Revista Brasileira de Economia, v. 43, p. 1-18, jan-jun 1989.

Salazar, Antônio; Carvalho, José Luis. Uma estimativa da taxa de câmbio de equilíbrio de livre comércio para o Brasil: 1960-1983. Revista Brasileira de Economia, v. 43, p. 1-18, jan-jun 1989.

Samuelson, Paul. Theoretical notes on trade problems. Review of Economics and Statistics 46, p. 145154, 1964. 